\title{
COMMENT
}

\section{Predicting childhood asthma development: are early life metabolite levels the philosopher's stone to unlock the puzzle?}

\author{
Antonia P. Popova ${ }^{1}$ and Marc B. Hershenson ${ }^{1}$ \\ Pediatric Research (2018) 84:593-594; https://doi.org/10.1038/s41390-018-0119-4
}

Asthma is the most common chronic lung disease of childhood, affecting approximately 6 million children in the United States. ${ }^{1}$ It is well recognized that asthma development depends on both genetic and environmental factors, originates early in life, and leads to chronic respiratory symptoms and airflow limitation. Although progress has been made in the care of children with asthma, including the reduction of adverse health outcomes and improvement of asthma control, ${ }^{1}$ we lack strategies to prevent asthma development or cure it. This knowledge gap stems from an incomplete understanding of the mechanisms leading to asthma development.

Models predicting childhood asthma development have focused on various prenatal and early postnatal biological factors. Among risk factors present in utero and at the time of delivery are a family history of atopic disease, black race, male sex, passive tobacco smoke exposure, and Caesarian section delivery (reviewed in ref. $^{2}$ ). A multitude of postnatal factors have also been associated with increased risk of asthma including respiratory viral infections, early life house-dust endotoxin exposure or colonization with specific bacteria, antibiotic use, obesity, allergen exposure, passive and active tobacco smoke exposure, trafficrelated pollutants, maternal depression, and familial stress. However, less is known about predictors of asthma development in healthy infants and children prior to the onset of symptoms. Several recent studies have examined maternal cytokine profiles during pregnancy, ${ }^{3}$ plasma chemokines, early life wheezingassociated respiratory infections with rhinovirus, ${ }^{4}$ and severe infantile eczema ${ }^{5}$ as predictors of childhood asthma development and indicate that a distinct phenotype is recognizable prior to the onset of asthma symptoms.

In this issue, Donovan et al. describe 1951 full-term healthy infants who were enrolled at birth in the Infant Susceptibility to Pulmonary Infections and Asthma Following RSV Exposure Study (INSPIRE). Participants were followed over a 3-year period to assess the association between newborn screening metabolite levels and wheezing at 1,2 , and 3 years of age ${ }^{6}$. After adjustment for asthma risk factors and potential confounders, the results demonstrate a strong association between several metabolites, specifically C10:1 and C18:2 acylcarnitines, and the number of wheezing episodes in childhood. These new findings highlight the potential of pre- and perinatal biochemical metabolic profile to predict recurrent wheezing and future asthma development.

Metabolomics is the study of the metabolite fingerprint, a library of small molecules within a cell type, tissue, organ, or whole organism. Providing a readout of complex biochemical processes occurring at the interface between genetic and environmental influences, metabolic profiling is an appealing method for the study of pathways determining childhood asthma development. By applying different targeted and untargeted metabolomics approaches on exhaled breath and exhaled breath condensate, asthmatic children have been distinguished from healthy children on the basis of oxidized and acetylated compounds ${ }^{7}$ and adenosine metabolites. ${ }^{8}$ In addition, exhaled breath volatile organic compounds have distinguished healthy children from asthmatics, ${ }^{9}$ wheezing from non-wheezing preschool children, ${ }^{10}$ and asthmatic children from transiently wheezing children. ${ }^{11}$ However, these studies compare established phenotypes rather than assessing the predictive potential and longitudinal changes in the metabolome along the course of development of a specific disease phenotype.

In this context, the study by Donovan et al. $^{6}$ investigated the association between targeted newborn screen metabolite levels in healthy infants and the number of subsequent episodes of wheezing per year in the first 3 years of life. The study found a significant association between two acylcarnitines (i.e., fatty acids) and the number of wheezing episodes in early childhood. The association for one metabolite, C10:1, remained significant after adjustment for other known risk factors for asthma and potential confounders, including gender, ethnicity, race, delivery type, maternal asthma diagnosis, maternal smoking, other household smokers, primary insurance enrollment, and daycare attendance in the first year of life. Thus, newborn C10:1 level was an independent predictor of future recurrent wheezing.

Acylcarnitines are intermediate metabolites of fatty acid oxidation that, among other functions, promote inflammation by activating pathogen recognition receptors and their downstream $\mathrm{NF}-\mathrm{KB}$ and $\mathrm{c}-\mathrm{Jun} \mathrm{N}$-terminal kinase signaling pathways leading to increased cytokine expression (reviewed in ref. ${ }^{12}$ ). Asthma risk alleles from the PDED3 and MED24 genes at the asthma susceptibility locus $17 q 21$ have been associated with increased concentrations of four phosphatidylcholines, consistent with the notion that lipid metabolites play a causative role in the development of asthma. ${ }^{13}$ However, whether altered lipid metabolism contributes to the pathogenesis of asthma or simply reflects metabolic changes resulting from asthma development remains unknown.

We should note that, while the primary outcome of the study by Donovan et al. is wheezing in early life, more than half of the children who wheeze in the first 3 years of life outgrow the symptoms by school age and do not develop persistent asthma. ${ }^{14}$ In addition, studies of early life viral infection have shown that community RSV infections are not associated with wheezing by 13

${ }^{1}$ Division of Pediatric Pulmonology, Department of Pediatrics and Communicable Diseases, Michigan Medicine, Ann Arbor, MI, USA

Correspondence: Antonia P. Popova (antoniap@umich.edu)

Received: 20 June 2018 Accepted: 3 July 2018

Published online: 23 July 2018 
years of age. ${ }^{4}$ Nevertheless, follow-up of the patients enrolled in the INSPIRE cohort will determine whether metabolite levels predict wheezing at older ages when asthma diagnosis can be more reliably ascertained. Perhaps in the future, pediatricians will order a simple blood test to help them with one of their most challenging tasks; prediction of asthma development in the school years.

\section{ADDITIONAL INFORMATION}

Competing interests: The authors declare no competing interests.

Publisher's note: Springer Nature remains neutral with regard to jurisdictional claims in published maps and institutional affiliations.

\section{REFERENCES}

1. Zahran, H. S., Bailey, C. M., Damon, S. A., Garbe, P. L. \& Breysse, P. N. Vital signs: asthma in children - United States, 2001-2016. Morb. Mortal. Wkly. Rep. 67, 149-155 (2018)

2. Guilbert, T. W., Mauger, D. T. \& Lemanske, R. F. Jr. Childhood asthma-predictive phenotype. J. Allergy Clin. Immunol. Pract. 2, 664-670 (2014).

3. Rothers, J., et al. Maternal cytokine profiles during pregnancy predict asthma in children of nonasthmatic mothers. Am. J. Respir. Cell Mol. Biol. (2018) https://doi. org/10.1165/rcmb.2017-04100C. PMID: 29863910.
4. Rubner, F. J. et al. Early life rhinovirus wheezing, allergic sensitization, and asthma risk at adolescence. J. Allergy Clin. Immunol. 139, 501-507 (2017).

5. Ekback, M. et al. Severe eczema in infancy can predict asthma development. A prospective study to the age of 10 years. PLOS ONE 9, e99609 (2014).

6. Donovan BM, Ryckman KK, Breheny PJ, et al. Association of newborn screening metabolites with risk of wheezing in childhood. Pediatr Res 2018

7. Carraro, S. et al. Metabolomics applied to exhaled breath condensate in childhood asthma. Am. J. Respir. Crit. Care. Med. 175, 986-990 (2007).

8. Esther, C. R. et al. Mass spectrometric analysis of biomarkers and dilution markers in exhaled breath condensate reveals elevated purines in asthma and cystic fibrosis. Am. J. Physiol. Lung Cell Mol. Physiol. 296, L987-L993 (2009).

9. Dallinga, J. W. et al. Volatile organic compounds in exhaled breath as a diagnostic tool for asthma in children. Clin. Exp. Allergy. 40, 68-76 (2010).

10. van de Kant, K. D. et al. Exhaled breath profiling in diagnosing wheezy preschool children. Eur. Respir. J. 41, 183-188 (2013).

11. Caldeira, M. et al. Allergic asthma exhaled breath metabolome: a challenge for comprehensive two-dimensional gas chromatography. J. Chromatogr. A 1254, 87-97 (2012).

12. McCoin, C. S., Knotts, T. A. \& Adams, S. H. Acylcarnitines-old actors auditioning for new roles in metabolic physiology. Nat. Rev. Endocrinol. 11, 617-625 (2015).

13. Ried, J. S. et al. Integrative genetic and metabolite profiling analysis suggests altered phosphatidylcholine metabolism in asthma. Allergy 68, 629-636 (2013).

14. Martinez, F. D. et al. Asthma and wheezing in the first six years of life. The Group Health Medical Associates. N. Engl. J. Med. 332, 133-138 (1995). 